\title{
Adaptive nonlinear control of single-phase to three-phase UPS system
}

\author{
M. Kissaoui ${ }^{1}$, A. Abouloifa ${ }^{2}$, F. Giri ${ }^{3}$, F.Z. Chaoui ${ }^{1}$ and Y. Abouelmahjoub ${ }^{1}$ \\ ${ }^{1}$ RCSLNL/LM2PI Lab, Mohammed V University Souissi, Rabat, Morocco \\ ${ }^{2}$ L.T.I Lab, FSBM, University HASSAN II Casablanca, Morocco \\ ${ }^{3}$ GREYC Lab, University of Caen Basse-Normandie, Caen, France
}

\begin{abstract}
This work deals with the problems of uninterruptible power supplies (UPS) based on the singlephase to three-phase converters built in two stages: an input bridge rectifier and an output three phase inverter. The two blocks are joined by a continuous intermediate bus. The objective of control is threefold: i) power factor correction "PFC", ii) generating a symmetrical three-phase system at the output even if the load is unknown, iii) regulating the DC bus voltage. The synthesis of controllers has been reached by two nonlinear techniques that are the sliding mode and adaptive backstepping control. The performances of regulators have been validated by numerical simulation in MATLAB / SIMULINK.
\end{abstract}

\section{Introduction}

Uninterruptible power supplies (UPS) play an important role in interfacing critical loads such as computers, communication systems, medical/life support systems, and industrial controls to the utility power grid. They are designed to provide clean and continuous power to the load under essentially any normal or abnormal utility power condition.

In this work, we focus on adaptive nonlinear control for single phase to three-phase UPS system figure1, which is based on the single-phase bridge rectifier, threephase inverter and an LC filter. All IGBT-diode switches with PWM control, to ensure control the DC component of the output of the rectifier, the correction of the power factor and generating a symmetric three phase system. To achieve these objectives three control loops are used in [1], [2].

The first inner loop is designed, using the sliding mode technique, to ensure PFC. The second inner loop is designed, using the adaptive backstepping approach, to generating a symmetrical three-phase system with a three-phase resistive load supposed unknown. The outerloop involves a PI regulator that regulates the DC bus voltage [3].

This paper is organized as follows: Section 2 is devoted to the description and modelling of single-phase to three-phase UPS System, the synthesis of regulators is developed in section 3. The closed loop performances are illustrated by simulation in Section 4. The conclusion ends the paper.

\section{System Descriptions and Modelling}

The single-phase to three-phase UPS system under study has the structure of Figure 1. At the same time, the two legs, IGBT-diode, works as a bridge rectifier and supply the DC-link bus with power. The other three legs work in inverter mode and feed the loads.

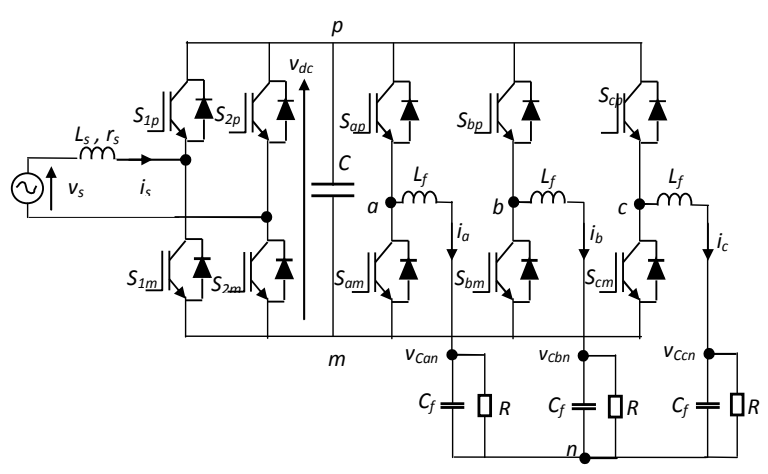

Fig .1. Single-phase to three-phase UPS system.

The switching functions and ${ }_{j}$ are defined by:

$$
\begin{aligned}
& =\left\{\begin{array}{c}
1 \quad \text { if } S_{1 p}, S_{2 m} \text { are } O N \text { and } S_{2 p}, S_{1 m} \text { are } O F F \\
1 \text { if } S_{2 p}, S_{1 m} \text { are } O N \text { and } S_{1 p}, S_{2 m} \text { are } O F F
\end{array}\right. \\
& j=\left\{\begin{array}{rr}
1 & \text { if } S_{j p} \text { is } O N \text { and } S_{j m} \text { is } O F F \\
1 & \text { if } S_{j m} \text { is } O N \text { and } S_{j p} \text { is } O F F
\end{array} \quad(j=a, b, c)\right.
\end{aligned}
$$


The average model, in $\mathrm{dq}$ frame, thus obtained is described by three subsystems as follow:

$L_{s} \frac{d x_{s}}{d t}=r_{s} x_{s}+v_{s} \quad u x_{d c}$

$\frac{d}{d t}\left(\begin{array}{l}x_{1 d} \\ x_{1 q}\end{array}\right)=\left(\begin{array}{ll}0 & \\ & 0\end{array}\right)\left(\begin{array}{l}x_{1 d} \\ x_{1 q}\end{array}\right)+\frac{1}{C_{f}}\left(\begin{array}{l}x_{2 d} \\ x_{2 q}\end{array}\right) \quad\left(\begin{array}{l}x_{1 d} \\ x_{1 q}\end{array}\right)$

$\frac{d}{d t}\left(\begin{array}{l}x_{2 d} \\ x_{2 q}\end{array}\right)=\left(\begin{array}{cc}0 & \\ & 0\end{array}\right)\left(\begin{array}{l}x_{2 d} \\ x_{2 q}\end{array}\right)+\frac{x_{d c}}{2 L_{f}}\left(\begin{array}{l}u_{d} \\ u_{q}\end{array}\right) \quad \frac{1}{L_{f}}\left(\begin{array}{l}x_{1 d} \\ x_{1 q}\end{array}\right)$

$C \frac{d x_{d c}}{d t}=u x_{s} \quad \frac{1}{2}\left(u_{d} x_{2 d}+u_{q} x_{2 q}\right)$

where the various state variables are the average values, over cutting periods, of the physical variables, see table1:

Table 1. The average values, over cutting periods, of the physical variables

\begin{tabular}{|c|c|}
\hline State variable & $\begin{array}{c}\text { Is the average values } \\
\text { of }\end{array}$ \\
\hline$x_{s}$ & $i_{s}$ \\
\hline$x_{d c}$ & $v_{d c}$ \\
\hline$x_{1 d}, x_{2 d}$ & $v_{C d}, i_{d}$ \\
\hline$x_{1 q}, x_{2 q}$ & $v_{C q}, i_{q}$ \\
\hline
\end{tabular}

Notice that the model of three subsystems (1), (2a-b) and (3) is clearly nonlinear and the mean values $u, u_{d}$ and $u_{q}$ of,$d_{d}$ and $q$ turn out to be the system control inputs. The parameter $=1 / R C_{f}$ is unknown, because the load resistance $R$ is assumed unknown.

\section{Controller Design}

The aim is to design a controller which is able to achieve three follows objectives:

PFC requirement: the current drawn by the single phase rectifier should be, in average, in phase with the grid voltage.

Output voltage regulation: the three-phase inverter must generate a symmetrical three-phase system despite the fact that the load resistance is unknown.

DC link voltage regulation: the DC component of the voltage must be stabilized to a desired reference voltage.

\subsection{PFC inner loop design}

Based on the first sub-system (1), The PFC objective is achieved by the regulator, using the sliding mode control [4], [5], that enforces the current $x_{s}$ to track a reference signal of the form $x_{s}^{*}=v_{s}(t)$, while is a positive real signal to be defined later.

Consider the sliding surface $S\left(x_{s}\right)$ defined by:

$S\left(x_{s}\right)=e_{i}=x_{s} \quad x_{s}^{*}$
The convergence condition is defined by the Lyapunov function $V=0.5 S^{2}\left(x_{s}\right)$ as follows:

$\dot{V}=S\left(x_{s}\right) \dot{S}\left(x_{s}\right)<0$

The equivalent component can be interpreted as the average value modulated, we obtain:

$u_{e q}=\frac{1}{x_{d c}}\left(r_{s} x_{s}+v_{s}\right)$

The non-linear component is determined to ensure the attraction of current to the sliding surface and satisfy the convergence condition described by (5), then:

$u_{n}=c_{i} \frac{L_{s}}{x_{d c}} \operatorname{sgn}\left[S\left(x_{s}\right)\right]$

The sliding mode control law is as follows:

$$
u=\frac{1}{x_{d c}}\left\{r_{s} x_{s}+v_{s}+c_{i} L_{s} \operatorname{sgn}\left[S\left(x_{s}\right)\right]\right\}
$$

\subsection{Three-phase system inner loop design}

The controller must force the three-phase system voltage of three-phase inverter DC / AC to track reference signals with the reference signals in dq frame follow:

$$
\left(\begin{array}{ll}
x_{1 d}^{*} & x_{1 q}^{*}
\end{array}\right)=\left(\begin{array}{ll}
0 & \sqrt{3 / 2} E
\end{array}\right)
$$

The synthesis technique used is known as the adaptive backstepping and is completed in two steps.

Step 1: Stabilization of the subsystem $\left(e_{1 d}, e_{1 q}, \sim\right)$

Consider the tracking error vector $\boldsymbol{E}_{1}$ defined by :

$E_{l}=\left(\begin{array}{l}e_{1 d} \\ e_{l q}\end{array}\right)=\left(\begin{array}{ll}x_{l d} & x_{l d}^{*} \\ x_{l q} & x_{l q}^{*}\end{array}\right)$

and its dynamics is given by:

$$
\dot{\boldsymbol{E}}_{1}=\left(\begin{array}{c}
\dot{\boldsymbol{e}}_{1 d} \\
\dot{\boldsymbol{e}}_{1 q}
\end{array}\right)=\left(\begin{array}{ccc}
\boldsymbol{x}_{1 q}+\boldsymbol{x}_{2 d} / \boldsymbol{C}_{f} & \boldsymbol{x}_{1 d} & \dot{\boldsymbol{x}}_{1 d}^{*} \\
\boldsymbol{x}_{1 d}+x_{2 q} / C_{f} & x_{1 q} & \dot{x}_{1 q}^{*}
\end{array}\right)
$$

We use the following Lyapunov candidate function:

$W_{1}=\frac{1}{2} e_{1 d}^{2}+\frac{1}{2} e_{l q}^{2}+\frac{1}{2} \sim_{2}$

Its derivative with respect to time is given by:

$$
\begin{aligned}
& \dot{W}_{l}=e_{I d}\left(x_{l q}+\frac{1}{C_{f}} x_{2 d} \quad \hat{x}_{1 d} \quad \dot{x}_{I d}^{*}\right) \\
& +e_{I q}\left(\begin{array}{lll}
x_{I d}+\frac{1}{C_{f}} x_{2 q} & \hat{x}_{1 q} & \dot{x}_{1 q}^{*}
\end{array}\right) \underline{1} \sim\left(\dot{\hat{\imath}}+\left(x_{I d} e_{I d}+x_{1 q} e_{I q}\right)\right)
\end{aligned}
$$

If $x_{2 d} / C_{f}={ }_{d}$ and $x_{2 q} / C_{f}={ }_{q}$ was our effective control, $d$ and $q$ are suitable stabilizing functions, it is sufficient to take:

$\left\{\begin{array}{l}{ }_{d}=c_{1 d} e_{1 d} \quad x_{1 q}+\hat{x_{1 d}}+\dot{x}_{1 d}^{*} \\ { }_{q}=c_{1 q} e_{1 q}+x_{1 d}+\hat{x_{1 q}}+\dot{x}_{1 q}^{*}\end{array}\right.$

where $\left(c_{1 d}, c_{1 q}\right)$ are positive constants of synthesis. 
As $x_{2 d} / C_{f}$ and $x_{2 q} / C_{f}$ are not the actual control inputs, a new vector error, denoted $E_{2}$, is introduced:

$\boldsymbol{E}_{2}=\left(\begin{array}{l}\boldsymbol{e}_{2 \boldsymbol{d}} \\ \boldsymbol{e}_{2 \boldsymbol{q}}\end{array}\right)=\left(\begin{array}{ll}\boldsymbol{x}_{2 \boldsymbol{d}} / \boldsymbol{C}_{\boldsymbol{f}} & \boldsymbol{d} \\ \boldsymbol{x}_{2 \boldsymbol{q}} / \boldsymbol{C}_{\boldsymbol{f}} & \boldsymbol{q}\end{array}\right)$

Then, equation (11) becomes, using (14) and (15):

$\dot{\boldsymbol{E}}_{l}=\left(\begin{array}{l}\dot{\boldsymbol{e}}_{1 d} \\ \dot{\boldsymbol{e}}_{l q}\end{array}\right)=\left(\begin{array}{cc}c_{1 d} \boldsymbol{e}_{1 d}+\boldsymbol{e}_{2 d} & \sim \boldsymbol{x}_{l d} \\ c_{l q} \boldsymbol{e}_{l q}+\boldsymbol{e}_{2 q} & \tilde{x}_{l q}\end{array}\right)$

Also, the derivative of Lyapunov function (13) becomes:

$$
\begin{gathered}
\dot{W}_{1}=c_{1 d} e_{1 d}^{2}+e_{1 d} e_{2 d} \quad c_{1 q} e_{1 q}^{2}+e_{1 q} e_{2 q} \\
\underline{1} \sim\left(\dot{i}+\left(x_{1 d} e_{1 d}+x_{1 q} e_{1 q}\right)\right)
\end{gathered}
$$

Step2: Stabilization of the subsystem

$\left(e_{1 d}, e_{1 q}, e_{2 d}, e_{2 q}, \sim\right)$

Time-derivation of $E_{2}$ gives, using (14) and (2b):

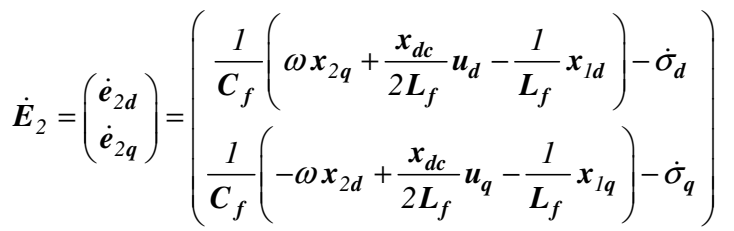

Derivatives of stabilizing functions can be written in the following form: ${ }_{d}={ }_{d}+{ }_{d} \sim$ and $\cdot{ }_{q}={ }_{q}+{ }_{q} \sim$

The actual control variables, namely, $u_{d}$ and $u_{q}$ appears for the first time (18). Let us consider the Lyapunov function.

$W_{2}=W_{1}+\frac{1}{2} e_{2 d}^{2}+\frac{1}{2} e_{2 q}^{2}$

Using (17), the time-derivative of $W_{2}$ can be rewritten as:

$$
\begin{aligned}
\dot{W}_{2} & =c_{1 d} e_{1 d}^{2} \quad c_{1 q} e_{1 q}^{2}+e_{2 d}\left(e_{1 d}+\dot{e}_{2 d}\right) \\
& +e_{2 q}\left(e_{1 q}+\dot{e}_{2 q}\right) \underline{1} \sim\left({ }^{\dot{i}}+\left(x_{1 d} e_{1 d}+x_{1 q} e_{1 q}\right)\right)
\end{aligned}
$$

This shows that, for the $\left(e_{1 d}, e_{1 q}, e_{2 d}, e_{2 q}, \sim\right)$-system to be globally asymptotically stable, it is sufficient to choose the control $u_{d}, u_{q}$ and $\dot{\sim}$ so that $\dot{W}_{2}=c_{1 d} e_{1 d}^{2} \quad c_{1 q} e_{1 q}^{2} \quad c_{2 d} e_{2 d}^{2} \quad c_{2 q} e_{2 q}^{2}$ which, due to (20), amounts to ensuring that:

$\dot{\boldsymbol{E}}_{2}=\left(\begin{array}{c}\dot{\boldsymbol{e}}_{2 \boldsymbol{d}} \\ \dot{\boldsymbol{e}}_{2 \boldsymbol{q}}\end{array}\right)=\left(\begin{array}{cccc}\boldsymbol{c}_{2 \boldsymbol{d}} \boldsymbol{e}_{2 \boldsymbol{d}} & \boldsymbol{e}_{1 \boldsymbol{d}} & \boldsymbol{d} \\ \boldsymbol{c}_{2 \boldsymbol{q}} \boldsymbol{e}_{2 \boldsymbol{q}} & \boldsymbol{e}_{1 \boldsymbol{q}} & & \boldsymbol{q}\end{array}\right)$

Comparing (18) and (21) yields the following backstepping control laws $u_{d}$ and $u_{q}$ : $u_{d}=\left[\begin{array}{ll}\left(2 L_{f} C_{f}\right) / x_{d c}\end{array}\right]\left[\begin{array}{lll}c_{2 d} e_{2 d} & e_{1 d} & x_{2 q} / C_{f}+x_{1 d} / L_{f} C_{f}+{ }_{d}\end{array}\right](22)$
$u_{q}=\left[\left(\begin{array}{lll}\left.2 L_{f} C_{f}\right) / x_{d k}\end{array}\right]\left[\begin{array}{lll}c_{2 q} e_{2 q} & e_{1 q}+ & x_{2 d} / C_{f}+x_{1 q} / L_{f} C_{f}+{ }_{q}\end{array}\right](23)\right.$

and adaptation law is:

$$
\dot{\sim}=\dot{i}=\left(x_{1 d} e_{1 d}+x_{1 q} e_{1 q}+{ }_{d} e_{2 d}+{ }_{q} e_{2 q}\right)
$$

\subsection{DC voltage outer loop design}

The aim of the outer loop is to generate a tuning law for the signal so that the output voltage $x_{d c}$ is steered to a given reference value $x_{d c}^{*}$. This is the subject of the following proposition.

Proposition: Consider the single-phase to three-phase described by (1, 2 and 3 ) augmented with the inner control laws defined by (8), (22) and (23), one has the following:

The squared-voltage $y=\left(x_{d c}\right)^{2}$ varies, in response to the tuning ratio, according to the following first-order time-varying linear equation:

$\dot{y}=f()+p(t)+q\left(x_{d q}\right)$

with

$f()=k_{0} \quad\left(\begin{array}{ll}1 & r_{s}\end{array}\right) ; p(t)=f() \cos (2 t)$

$q\left(x_{d q}\right)=\frac{2}{C}\left(x_{1 d} x_{2 d}+x_{1 q} x_{2 q}+{ }_{d} x_{2 d}+{ }_{q} x_{2 q}\right)$

where $\quad k_{0}=E^{2} / C$

Proof: it is identical to a similar result in [6]

The current problem is to design a suitable control law so that the square of the voltage $y=\left(x_{d c}\right)^{2}$ follows a reference signal given $y^{*}=\left(x_{d c}^{*}\right)^{2}$. As the term disruptive $p(t)$ in (25), is periodic with zero mean and a control law PI with compensation for nonlinearity $q\left(x_{d q}\right)$, should suffice:

$f()=c_{3} e_{3}+c_{4} e_{4} \quad q\left(x_{d q}\right)+y^{*}$

with $e_{3}=y^{*} y \quad ; \quad e_{4}=\int_{0}^{t} e_{3} d$

Substituting equation (25) in (26), we obtain the equations errors $e_{3}$ and $e_{4}$.

$\left\{\begin{array}{c}\dot{e}_{3}=c_{3} e_{3} \quad c_{4} e_{4} \quad p(t) \\ \dot{e}_{4}=e_{3}\end{array}\right.$

At this point, the regulator parameters $\left(c_{3}, c_{4}\right)$ are any positive real constants. The actual control signal can be easily obtained from (26) using the fact that $f^{l}($. exists. 


\section{Numerical Simulations}

The performances of proposed controllers are validated by simulation in MATLAB/SIMULINK environment. The parameters of the controlled system are given in the table 2. The main voltage is fixed at its nominal value $v_{s}(t)=E \sin (t)$. Figures 2 to 5 show the simulation results of uninterrupted power system based on the single-phase to three-phase under the effect of a load change when the reference DC bus voltage is set at $1600 \mathrm{~V}$. The variable load $\mathrm{R}$ is periodic with period $0.6 \mathrm{~s}$ alternates between 10 and 20 in each half period.

Table 2. System parameters controlled.

\begin{tabular}{|c|c|c|}
\hline Parameters & Symbol & Values \\
\hline Network & $E, f$ & $220 \sqrt{2} \mathrm{~V}, 50 \mathrm{~Hz}$ \\
\hline Rectifier & $L_{s}$ & $2 \mathrm{mH}$ \\
& $r_{s}$ & $30 \mathrm{~m}$ \\
\hline Dc Bus & $C$ & $47 \mathrm{mF}$ \\
\hline Three-phase inverter & $L_{f}$ & $100 \mathrm{mH}$ \\
& $C_{f}$ & $100 \mathrm{~F}$ \\
\hline PFC Regulator & $c_{i}$ & $210^{3}$ \\
\hline Three phase system & $c_{1 d}=c_{1 q}$ & 1000 \\
Regulator & $c_{2 d}=c_{2 q}$ & $10^{4}$ \\
& & $10^{6}$ \\
\hline DC voltage & $c_{3}$ & $210^{5}$ \\
Regulator & $c_{4}$ & $610^{4}$ \\
\hline
\end{tabular}

In Figure 2, we see that the current $i_{s}$ and the input voltage $v_{s}$ are sinusoidal and in phase with the current amplitude change inversely proportional to the change of the load. This shows that the correction of the power factor is well established. Figure 3 shows the evolution of the output phase voltages $v_{C a n}, v_{C b n}$ and $v_{C c n}$ of the three phase inverter which is a three-phase system that provides balance even if the load changes. This is because the estimated converges rapidly to its true value (Figure 4). Finally, Figure 5 shows that the DC bus voltage $x_{d c}$ perfectly follows (in average) its reference.

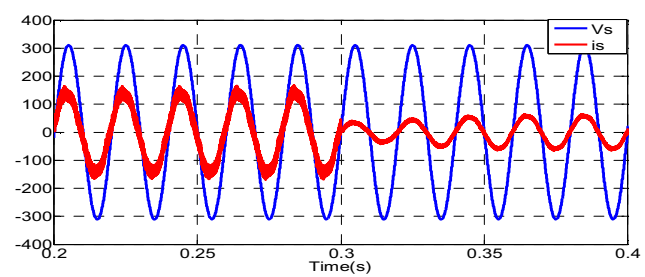

Fig. 2. Input voltage $v_{s}$ and input current $i_{s}$

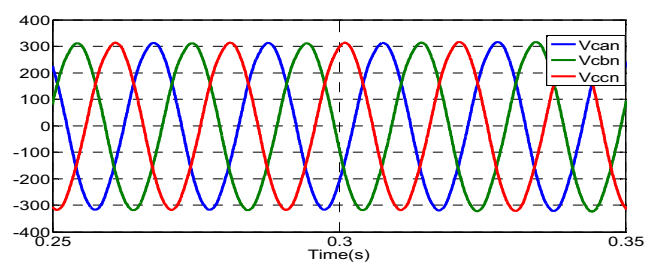

Fig. 3. Phase output voltages $v_{C a n}, v_{C b n}$ and $v_{C c n}$

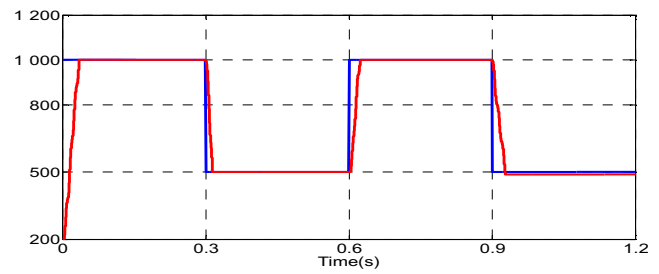

Fig. 4. Estimated parameter ${ }^{\wedge}$ (red) in the presence of (blue)

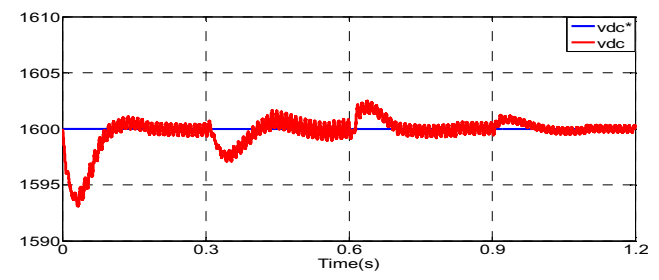

Fig. 5. DC bus voltage $x_{d c}$ and reference $x_{d c}^{*}$

\section{Conclusion}

In this paper, an adaptive nonlinear controller is proposed for the single-phase to three-phase UPS System used in power systems without interruption. It has been formally established that the obtained controller meets its objectives such as: i) unity input power factor PFC feature is enabled, ii) high-quality sinusoidal output voltages, even with a unknown resistive load, iii) regulation of the DC bus voltage, and iv) excellent transient characteristics and stability.

\section{References}

[1] F. Giri, A. Abouloifa, I. Lachkar, F.Z. Chaoui, Nonlinear Control of PWM AC/DC Boost Rectifiers: Theoretical Analysis of Closed-Loop Performances, 17th Proceedings of the IFAC World Congress, Seoul, Korea, (2008).

[2] J.Y. Choi, J. Farrell, Observer-based Backstepping Control Using Online Approximation, American Control Conference, 5, 3646-3650, (2000).

[3] R. Ghosh, G. Narayanan, A Simple Analog Controller for Single-Phase Half-Bridge Rectifier and its Application to Transformerless UPS, IEEE IISC, (2005).

[4] S-El-M. Ardjoun, M. Abid, A-G. Aissaoui, A. Naceri, A robust fuzzy sliding mode control applied to the double fed induction machine, International Journal Of Circuits, Systems And Signal Processing 4, 5, pp. 315-321, NAUN, USA, (2011).

[5] S. Chattopadhyay, V. Ramanaryanan, A single-resetintegrator-based implementation of lince-current-shapping controller for high-power-factor operation of flyback rectifier, IEEE Trans. on industry application 38, 490-499, March-April (2002).

[6] M. Kissaoui, A. Abouloifa, F. Giri, F.Z. Chaoui, Y. Abouelmahjoub, Nonlinear Control of New Single-Phase to Three-Phase Hybrid UPS System, International Conference on Control, Engineering \& Information Technology (CEIT'14), 6, 109-117 (2014). 